\title{
Self-Averaging and Ergodicity of Subdiffusion in Quenched Random Media
}

\author{
Marco Dentz \\ Spanish National Research Council (IDAEA-CSIC), 08034 Barcelona, Spain* \\ Anna Russian and Philippe Gouze \\ Géosciences, Université de Montpellier 2, CNRS, Montpellier, France
}

(Dated: January 8, 2016)

\begin{abstract}
We study the self-averaging properties and ergodicity of the mean square displacement $m(t)$ of particles diffusing in $d$ dimensional quenched random environments which give rise to subdiffusive average motion. These properties are investigated in terms of the sample to sample fluctuations as measured by the variance of $m(t)$. We find that $m(t)$ is not self-averaging for $d<2$ due to the inefficient disorder sampling by random motion in a single realization. For $d \geq 2$ in contrast, the efficient sampling of heterogeneity by the space random walk renders $m(t)$ self-averaging and thus ergodic. This is remarkable because the average particle motion in $d>2$ obeys a CTRW, which by itself displays weak ergodicity breaking. This paradox is resolved by the observation that the CTRW as an average model does not reflect the disorder sampling by random motion in a single medium realization.
\end{abstract}

Anomalous diffusion has been ubiquitously observed in quenched random environments $[1-3]$ for processes as diverse as the motion of proteins in living cells [4-6], the transmission of light in optical media [7], the motion of charge carriers in amorphous semi-conductors [8], and particle and solute transport in heterogeneous porous media [9-11]. The ergodicity of stochastic particle motions $[12,13]$ and the self-averaging properties of the mean square displacement $m(t)$ [1] are central aspects for the understanding and prediction of diffusion processes in random media because they measure how well the diffusion behavior in a single disorder realization can be described by the ensemble dynamics.

In many quenched disordered systems, the ensemble particle motions obey continuous time random walk (CTRW) dynamics [10, 13-15] for the particle positions $\mathbf{x}_{n}$ and times $t_{n}$,

$$
\mathbf{x}_{n+1}=\mathbf{x}_{n}+\boldsymbol{\zeta}_{n}, \quad t_{n+1}=t_{n}+\tau_{n},
$$

which rely on the independence of subsequent space and time increments $\boldsymbol{\zeta}_{n}$ and $\tau_{n}$ (annealed disorder). Diffusion in $d>2$ dimensional random media follows in average a CTRW because particles sample the disorder in such a way that the average number of newly explored sites increases with the number of random walk steps [1,2]. For $d \leq 2$, correlations induced by a lower sampling efficiency lead to different, but CTRW like average behaviors.

Spurred by results from single particle tracking for diffusion in complex media, the question of ergodicity has been studied within the CTRW approach $[4,13,16-$ 18], and CTRW like models that describe the ensemble diffusion behaviors in quenched disorder models for $d \leq 2[6,19,20]$. As these approaches describe ensemble particle motions, they do not conserve information on the underlying individual disorder realizations. Therefore, in principle they do not provide a way to quantify fluctuations between disorder realizations. This problem is dealt with in the above cited works by identifying a realization of the transition time process $\left\{\tau_{n}\right\}$ with a disorder realization. This identification however assumes that all particles experience exactly the same disorder sequence in a single disorder realization. Thus, it does not account for disorder sampling due to the diffusive random motion, which is the mechanism that leads to CTRW ensemble dynamics in $d>2$, and governs the dynamics of the sample to sample fluctuations between realizations. The above cited works do not account for these key mechanisms when they conclude that the time averaged mean square displacement is weakly non-ergodic.

In this Letter we study the self-averaging properties and ergodicity of the mean square displacement in $d-$ dimensional quenched random environments. Starting from a stochastic disorder model, we clearly distinguish between the ensemble particle motion and the diffusive random motion in single medium realizations. This allows us to explicitly quantify the disorder sampling through this diffusive random motion, which is the mechanism that governs the fluctuations of the diffusion behaviors between medium realizations.

Diffusion in Quenched Disorder. - Diffusive particle motion in a single realization of a quenched random medium can be described by the Langevin equation

$$
\mathrm{d} \mathbf{x}(t)=\sqrt{2 D[\mathbf{x}(t)] \mathrm{d} t} \boldsymbol{\xi}(t),
$$

where we use the Ito interpretation. The Gaussian white noise has zero mean and covariance $\left\langle\xi_{i}(t) \xi_{j}\left(t^{\prime}\right)\right\rangle=$ $\delta_{i j} \delta\left(t-t^{\prime}\right)$, where the angular brackets denote the noise average. The particle distribution is given in terms of the trajectories $\mathbf{x}(t)$ by $p(\mathbf{x}, t)=\langle\delta[\mathbf{x}-\mathbf{x}(t)]\rangle$. The diffusion coefficient is set to $D(\mathbf{x})=\kappa \theta(\mathbf{x})^{-1}$, where $\kappa$ is the constant molecular diffusion coefficient. The quenched random mobility $\theta(\mathbf{x})$ represents the medium heterogeneity and quantifies particle retention due to physical and chemical interactions with the medium. The discrete in 
space version of this model is equivalent to the quenched random trap model [1] as outlined below. We investigate the mean-square displacement $m(t)=\left\langle\mathbf{x}(t)^{2}\right\rangle$ and focus on its ergodicity and self-averaging properties.

Single Disorder Realization. - In a single disorder realization, $m(t)$ is given by [21]

$$
m(t)=2 \kappa\langle s(t)\rangle d
$$

where we introduced the process $\mathrm{d} s=\theta[\mathbf{x}(t)]^{-1} \mathrm{~d} t$, which transforms the Langevin equation (2) to

$$
\mathrm{d} \mathbf{x}(s)=\sqrt{2 \kappa \mathrm{d} s} \boldsymbol{\xi}(s), \quad \mathrm{d} t(s)=\theta[\mathbf{x}(s)] \mathrm{d} s .
$$

In order to probe the ergodicity of $m(t)$, we consider the time averaged mean square displacement [17]

$$
m_{\Delta}(t)=\frac{1}{t-\Delta} \int_{0}^{t-\Delta} \mathrm{d} t^{\prime}\left[\mathbf{x}\left(t^{\prime}+\Delta\right)-\mathbf{x}\left(t^{\prime}\right)\right]^{2},
$$

for lag times $\Delta \ll t$. The relative variance of $m_{\Delta}(t)$ for $\Delta$ with respect to the noise or disorder averages is an indicator for its ergodicity [17]. In a single disorder realization, the variance of $m_{\Delta}(t)$ with respect to the noise average is, $\left\langle m_{\Delta}(t)^{2}\right\rangle-\left\langle m_{\Delta}(t)\right\rangle^{2}=0$ [21]. Diffusion is of course ergodic in the sense of Refs. [13, 17]. Note that the noise average of $m_{\Delta}(t)$ is given by $\left\langle m_{\Delta}(t)\right\rangle=$ $m(t) \Delta / t$, which provides an explicit relation between the time and noise average mean square displacements [21].

Sample to Sample Fluctuations. - The sample to sample fluctuations of the mean square displacement are probed by the relative variance

$$
\Sigma(t)=\frac{\sigma_{m}^{2}(t)}{\bar{m}(t)^{2}}, \quad \sigma_{m}^{2}(t)=\overline{m(t)^{2}}-\bar{m}(t)^{2}
$$

with $\sigma_{m}^{2}(t)$ the variance of $m(t)$ between disorder realizations. The overbar in the following denotes the disorder average. If $\lim _{t \rightarrow \infty} \Sigma(t)=0, m(t)$ is referred to as selfaveraging, otherwise as non self-averaging [1]. Note that the relative variance of $\left\langle m_{\Delta}(t)\right\rangle$ is identical to $\Sigma(t)$. Thus, $\Sigma(t)$ may be also considered a measure of ergodicity in the sense of Refs. [13, 17].

Coarse Graining. - We consider a stationary and isotropic random field $\theta(\mathbf{x})$, which is characterized by a characteristic correlation scale $\ell$. We extract the large scale particle dynamics through coarse graining. Thus, we note that on observation scales much larger than $\ell$, the organization of $\theta(\mathbf{x})$ may be represented by the medium illustrated in Figure 1 for $d=2$. The point values of $\theta(\mathbf{x})$ are constant within the distance $\ell$ and distributed randomly according to $p_{\theta}(\theta)$.

The coarse grained particle motions are obtained from the Langevin equation (4) as

$$
\mathbf{x}_{n+1}=\mathbf{x}_{n}+\ell \boldsymbol{\eta}_{n+1}, \quad t_{n+1}=t_{n}+\theta\left(\mathbf{x}_{n}\right) \hat{\tau},
$$

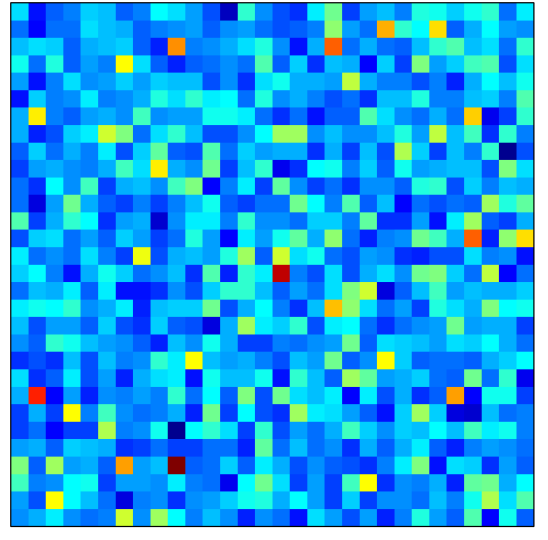

FIG. 1: Section $(30 \times 30$ pixels $)$ of a realization of a quenched random medium organized in equally sized pixels. To each pixel a random value of mobility $\theta$ is assigned from the Pareto distribution $p_{\theta}(\theta)=\beta \theta^{-1-\beta}$ for $\theta>1$. The numerical MonteCarlo simulations based on (7) for $\mathbf{x}_{0}=\mathbf{0}$ use $10^{6}$ particles in $10^{3}$ disorder realizations and domain sizes of $(d=3) 1.25 \times 10^{8}$ voxels, $(d=2) 10^{6}$ pixels and $(d=1) 1.2 \times 10^{3}$ pixels.

where $\hat{\tau}$ is the first passage time to the boundaries of a region of size $\ell$ by regular diffusion. We approximate its distribution here by the exponential $\psi_{0}(\hat{\tau})=$ $\tau_{\kappa}^{-1} \exp \left(-\hat{\tau} / \tau_{\kappa}\right)$ with the characteristic diffusion time $\tau_{\kappa}=\ell^{2} /(2 \kappa)[10,22,23]$. The unit random vector $\boldsymbol{\eta}$ is distributed according to $\psi_{\eta}(\boldsymbol{\eta})$ with mean $\langle\boldsymbol{\eta}\rangle=\mathbf{0}$ and $\left\langle\eta_{i} \eta_{j}\right\rangle=\delta_{i j}$. According to (7) particles describe an unbiased space random walk characterized by a transition length given by the characteristic heterogeneity scale. The transition time over the length $\ell$ is given by $\tau\left(\mathbf{x}_{n}\right)=\theta\left(\mathbf{x}_{n}\right) \hat{\tau}$, which, through its dependence on $\theta\left(\mathbf{x}_{n}\right)$ is a quenched random variable. Note that the quenched disorder model under consideration is equivalent to the quenched random trap model [1].

The distribution $\psi(\tau)$ of transition times $\tau$ is directly related to the distribution of the quenched disorder represented by $\theta(\mathbf{x})$ as

$$
\psi(\tau)=\int_{0}^{\infty} \mathrm{d} \theta \theta^{-1} p_{\theta}(\theta) \psi_{0}(\tau / \theta) .
$$

We consider a heavy tailed distribution of $\theta$ with $p_{\theta}(\theta) \propto$ $\theta^{-1-\beta}$, which gives for the transition time distribution $\psi(\tau) \propto \tau^{-1-\beta}$ for $\tau \ll \tau_{\kappa}$. We focus here on the range $0<\beta<1$, for which subdiffusive behavior is observed [1].

The governing equations (7) describe a time domain random walk in a quenched random environment, whose space-time trajectory is given by

$$
\mathbf{x}_{n}=\sum_{k=1}^{n} \ell \boldsymbol{\eta}_{k}, \quad t_{n}=\sum_{k=1}^{n} \tau\left(\mathbf{x}_{k-1}\right) .
$$

The particle density is given by $p(\mathbf{x}, t)=\left\langle\delta\left(\mathbf{x}-\mathbf{x}_{n_{t}}\right)\right\rangle$, where $n_{t}=\sup \left(n \mid t_{n} \leq t\right)$; the angular brackets denote 
the noise average over all particles. The particle density $p(\mathbf{x}, t)$ in a single disorder realization can be written as $[21]$

$$
p(\mathbf{x}, t)=\sum_{n=0}^{\infty}\left\langle\delta\left(\mathbf{x}-\mathbf{x}_{n}\right) \mathbb{I}\left(t_{n} \leq t<t_{n+1}\right)\right\rangle,
$$

where the indicator function $\mathbb{I}\left(t_{n} \leq t<t_{n+1}\right)$ is 1 if the statement in the parenthesis is true and 0 else. The mean square displacement (3) in a single disorder realization is now given terms of $n_{t}$ as $m(t)=d \ell^{2}\left\langle n_{t}\right\rangle$ [19, 21], which can be expressed by using (10) as

$$
m(t)=d \ell^{2} \sum_{n=0}^{\infty} n\left\langle\mathbb{I}\left[t_{n} \leq t<t_{n+1}\right]\right\rangle .
$$

As we will see in the following, the noise average denoted by the angular brackets plays a vital role for the quantification of the sample to sample fluctuations of $m(t)$.

Disorder Sampling and Noise Average. - In order to determine the average particle dynamics and its fluctuations, we need to quantify the ensemble statistics of (9) and in particular the impact of the noise on the disorder sampling in single disorder realizations. To this end, we recall the average number $S_{n}$ of distinct sites visited during the $d$-dimensional random walk (7). It is well known that $S_{n} \sim n^{d / 2}$ for $d<2, S_{n}=n / \ln (n)$ in $d=2$ and $S_{n} \sim n$ for $d>2$ [1]. Thus, the series of transition times $\left\{\tau_{k}\right\}_{k=1}^{n}$ with $\tau_{k} \equiv \tau\left(\mathbf{x}_{k-1}\right)$ can be reorganized into $S_{n}$ blocks of independent transition times $\tau_{i}$. Each block contains $n / S_{n}$ identical members. Thus, we represent now the particle motion in a single disorder realization in terms of the renormalized surrogate trajectories

$$
\mathbf{x}_{n}=\sum_{i=1}^{n} \ell \boldsymbol{\eta}_{i}, \quad t_{n}=\sum_{i=1}^{S_{n}} \frac{n}{S_{n}} \tau_{i},
$$

where the $\tau_{i}$ are independent increments distributed according to (8).

In a single disorder realization, the spectrum of $\tau_{i}$ values that is sampled by the particles is limited by the diffusive random motion. In fact, the average number of different sites explored by the ensemble of random walkers is $(2 n)^{d / 2}$, which quantifies the volume covered by the space random walk, while the number of distinct sites visited after $n$ random walk steps, that is, the number of independent $\tau_{i}$ in (12) is given by $S_{n}$. In other words, the average number of available sites after $n$ steps to form $t_{n}$ is $(2 n)^{d / 2}$ while the average number of sites that contribute to $t_{n}$ is $S_{n}$. Thus, the number $R_{n}$ of independent replica of $t_{n}$ is given by

$$
R_{n}=\frac{(2 n)^{d / 2}}{S_{n}} .
$$

The $R_{n}$ can be seen as measure for the efficiency by which the random walk samples the disorder. Thus, the mean square displacement (11) in a single disorder realization can be approximated in terms of the renormalized particle trajectories (12) as [21]

$$
m(t) \approx d \ell^{2} \sum_{n=0}^{\infty} n \frac{1}{R_{n}} \sum_{i=1}^{R_{n}} \mathbb{I}\left[t_{n}^{(i)} \leq t<t_{n+1}^{(i)}\right]
$$

The behavior of $R_{n}$ with $n$ determines the selfaveraging behavior with respect to the ensemble expectations of the particle trajectory and in particular the mean square displacement, as discussed below. It is intuitively clear that the sampling efficiency in $d<2$ is rather low. In fact, the number of independent replica is $R_{n} \sim 1$, i.e., all the particles see in average the same disorder. In $d=2$ dimensions, $R_{n} \sim \ln (n)$, and in $d=3$, $R_{n} \sim \sqrt{n}$, i.e., the number of independent replica, and thus the part of the disorder spectrum sampled increases with the number of random walk steps.

Note that some authors [19] ignore the noise average and identify the mean square displacement in a single disorder realization with $m(t) \equiv d \ell^{2} n_{t}$. This implies that a trajectory $t_{n}$ in (12) is identified with a disorder realization, which is justified for $d<2$. For $d \geq 2$, however, it does not reflect the actual sampling process.

Average Mean Square Displacement. - The behaviors of the mean square displacement have been well known in the literature [1]. We provide here an alternative derivation and a brief summary of the scaling behaviors.

The disorder averaged mean square displacement $\bar{m}(t)$ is obtained by the ensemble averages of either (11) or (14)

$$
\bar{m}(t)=d \ell^{2} \sum_{n=0}^{\infty} n \overline{\mathbb{I}\left(t_{n} \leq t<t_{n+1}\right)} .
$$

It can be expanded to the explicit expression [21]

$$
\bar{m}(t) \approx d \ell^{2} \int_{0}^{\infty} \mathrm{d} n n \frac{t}{\alpha_{n}} \frac{\mathrm{d} \ln \left(\alpha_{n}\right)}{\mathrm{d} n} f_{\beta}\left(t / \alpha_{n}\right),
$$

where we defined $\alpha_{n}=n S_{n}^{1 / \beta-1}$. The function $f_{\beta}(t)$ denotes the one-sided stable distribution with stability parameter $\beta$, whose Laplace transform [24] is given by $f_{\beta}(\lambda)=\exp \left(-a_{\beta} \lambda^{\beta}\right)$. Laplace transformed quantities are distinguished from their time-space equivalents through the argument. Using $S_{n} \sim n^{d / 2}$ for $d<2$ in (16) and rescaling the integration variable give the well known behavior for the average mean square displacement $[1,21]$

$$
\bar{m}(t) \propto t^{\frac{2 \beta}{2 \beta-d \beta+d}} .
$$

For $d=2$, we set $S_{n} \sim n / \ln (n)$ and $d>2, S_{n} \sim n$, which gives the scalings

$$
\bar{m}(t) \propto t^{\beta} \ln (t)^{1-\beta}, \quad \bar{m}(t) \propto t^{\beta},
$$



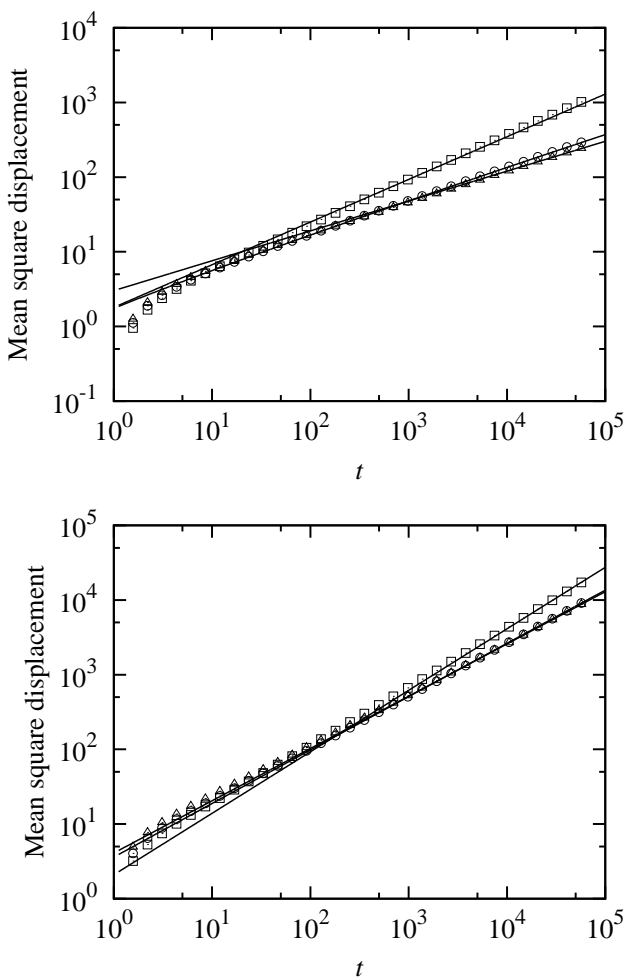

FIG. 2: Average mean-squared displacement from numerical Monte-Carlo simulations based on (7), for (squares) $d=1$, (circles) $d=2$ and (triangles) $d=3$ dimensions for (top figure) $\beta=2 / 5$ and (bottom figure) $\beta=7 / 10$. The lines represent the scaling behaviors (17) and (18). respectively. Figure (2) shows the average mean square displacement from Monte-Carlo simulations in $10^{3}$ realizations of $d=1, d=2$ and $d=3$ versions of the random medium illustrated in Figure 1. The analytical scalings (17) and (18) are fully confirmed by the numerical simulations.

It has been frequently pointed out [1] that diffusion in quenched random media describes in ensemble average a CTRW in $d>2$ spatial dimensions. This means, the ensemble particle motions can be described in terms of an annealed disorder process. This is not valid for other transport aspects. In particular, the sample to sample fluctuations of the mean square displacement reflects clearly the quenched nature of the underlying disorder as detailed in the following.

Self-Averaging and Ergodicity. - As outlined above, the sample to sample fluctuations of $m(t)$ are measured by the variance $\sigma_{m}^{2}(t)$ defined in (6). Based on expressions (14) for a single realization and (15) for the ensemble average mean square displacement, we derive for the variance $\sigma_{m}^{2}(t)[21]$

$$
\begin{aligned}
\sigma_{m}^{2}(t) & \approx d^{2} \ell^{4} \int_{0}^{\infty} \mathrm{d} n \frac{n^{2}}{R_{n}} \frac{t}{\alpha_{n}} \frac{\mathrm{d} \ln \left(\alpha_{n}\right)}{\mathrm{d} n} f_{\beta}\left(t / \alpha_{n}\right)-2 d^{2} \ell^{4} \int_{0}^{\infty} d n \frac{n}{R_{n}} \frac{t}{\alpha_{n}} \frac{\mathrm{d} \ln \left(\alpha_{n}\right)}{\mathrm{d} n} f_{\beta}\left(t / \alpha_{n}\right) \int_{0}^{n} \mathrm{~d} k k \frac{t}{\alpha_{k}} \frac{\mathrm{d} \ln \left(\alpha_{k}\right)}{\mathrm{d} k} f_{\beta}\left(t / \alpha_{k}\right) \\
& -d^{2} \ell^{4} \int_{0}^{\infty} \mathrm{d} n \frac{n^{2}}{R_{n}}\left[\frac{t}{\alpha_{n}} \frac{\mathrm{d} \ln \left(\alpha_{n}\right)}{\mathrm{d} n} f_{\beta}\left(t / \alpha_{n}\right)\right]^{2} .
\end{aligned}
$$

The temporal scalings of the different terms contributing to the variance are extracted in the same way as done for the average mean square displacement. Recall that $\alpha_{n}=n S_{n}^{1 / \beta-1}$ and $R_{n}=(2 n)^{d / 2} / S_{n}$. Inserting the respective expressions of $S_{n}$ for $d<2, d=2$ and $d>2$ in (19) and rescaling of the integration variables gives the temporal scaling of the variance. Specifically, we find that the third term on the right side is subleading, while the first two terms determine the leading asymptotic time behavior [21].

In $d<2$ dimensions, we find

$$
\sigma_{m}^{2}(t) \propto \bar{m}(t)^{2} \propto t^{\frac{4 \beta}{2 \beta-d \beta+d}} .
$$

This implies that dispersion is not self-averaging in $d<2$ because the relative variance $\Sigma(t)$ asymptotes towards a constant in the limit of $t \rightarrow \infty, \lim _{t \rightarrow \infty} \Sigma(t) \neq 0$. For $d \geq 2$ dimensions this is different. For $d=2$, we obtain for $\sigma_{m}^{2}(t)$ the scaling

$$
\sigma_{m}^{2}(t) \propto t^{2 \beta} \ln (t)^{1-2 \beta}
$$

Thus, the relative variance decays to zero logarithmically in the limit of infinite times, $\Sigma(t) \propto 1 / \ln (t)$ because $\bar{m}(t)$ behaves as (18). For $d>2$ dimensions we find the scaling

$$
\sigma_{m}^{2}(t) \propto t^{\beta(3-d / 2)} .
$$



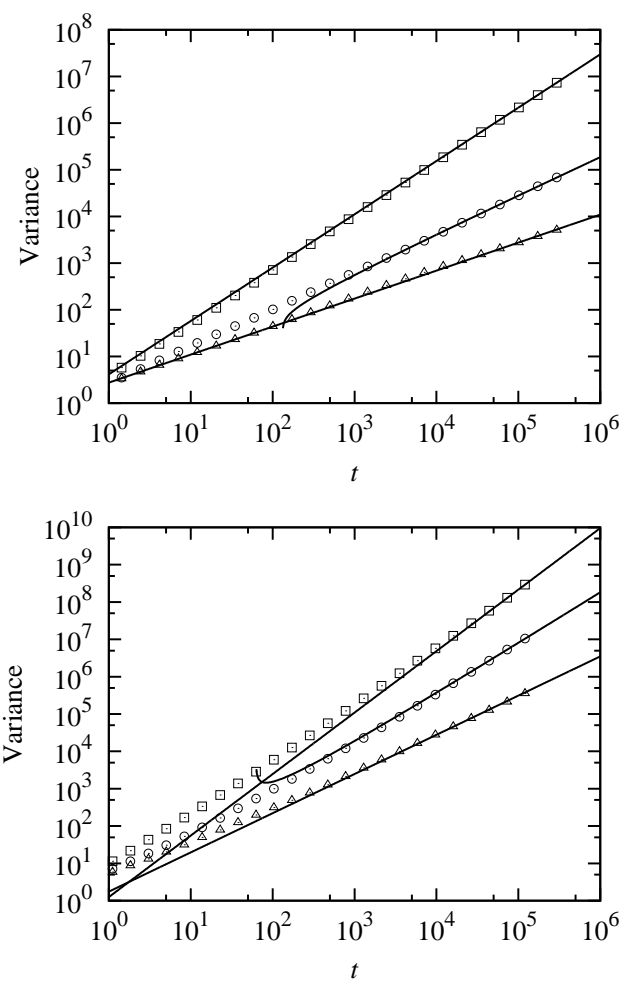

FIG. 3: Variances of the mean-squared displacement from numerical Monte-Carlo simulations based on (7), for (squares) $d=1$, (circles) $d=2$ and (triangles) $d=3$ dimensions for (top figure) $\beta=2 / 5$ and (bottom figure) $\beta=7 / 10$. The lines represent the scaling behaviors (20), (21) and (22).

The relative variance then scales as $\Sigma(t) \propto t^{\beta(1-d / 2)}$ according to (18). It decays faster as the dimensionality of space increases. Diffusion is self-averaging for $d \geq 2$ and thus ergodic in the sense of Refs. [13, 17]. Figure 3 illustrates the temporal evolution of $\sigma_{m}^{2}(t)$ sampled from numerical Monte-Carlo simulations over $10^{3}$ realizations of the quenched random medium illustrated in Figure 1. The asymptotic behavior of the numerical data confirms the derived scaling behaviors (20)-(22).

In conclusion, we find that the mean square displacement for diffusion in a quenched random medium is not self-averaging or ergodic for $d<2$ due to the inefficient spatial sampling of heterogeneity. In fact all the particles explore in a given realization in average the same disorder sequence. Such a sampling behavior is present in the CTRW (1) if a time trajectory $t_{n}$ is interpreted as a single disorder realization [17, 19]. In $d \geq 2$ on the other hand, the mean square displacement is self-averaging and thus ergodic due to the efficient heterogeneity sampling by the diffusive random motion. This is a remarkable finding because the ensemble particle motion in $d>2$ actually follows the CTRW (1). As discussed in the Introduction, the CTRW displays weak ergodicity breaking for the mean square displacement $[6,13,17]$ because it simulates a low sampling efficiency. Thus, while the average particle motion follows a CTRW for $d>2$, the quenched nature of the underlying disorder and the sampling efficiency of the diffusive random motion lead to a very different fluctuation behavior. These results emphasize the importance of the sound understanding of the fluctuation mechanisms underlying observed anomalous behaviors, and shed new light on the interpretation and prediction of particle movements and solute transport in quenched random environments.

M.D. acknowledges the funding from the European Research Council through the project MHetScale (Grant agreement no. 617511).

* Electronic address: marco.dentz@csic.es

[1] J. P. Bouchaud and A. Georges, Phys. Rep. 195, 127 (1990).

[2] S. Havlin and D. Ben-Avraham, Adv. Phys. 51, 187 (2002).

[3] I. M. Sokolov, Soft Matter 8, 9043 (2012).

[4] E. Barkai, Y. Garini, and R. Metzler, Phys. Today, No. 8, 65, 29 (2012). 8, 29 (2012).

[5] F. Höfling and T. Franosch, Rep. Prog. Phys. 76, 046602 (2013).

[6] P. Massignan, C. Manzo, J. A. Torreno-Pina, M. F. García-Parajo, M. Lewenstein, and J. Lapeyre, G. J., Phys. Rev. Lett. 112, 150603 (2014).

[7] P. Barthelemy, J. Bertolotti, and D. S. Wiersma, Nature 453, 495 (2008).

[8] H. Scher and M. Lax, Phys. Rev. B 7, 4491 (1973).

[9] B. Berkowitz and H. Scher, Phys. Rev. Lett. 79, 4038 (1997)

[10] B. Berkowitz, A. Cortis, M. Dentz, and H. Scher, Rev. Geophys. 44, RG2003 (2006).

[11] T. Le Borgne, M. Dentz, and J. Carrera, Phys. Rev. Lett. 101, 090601 (2008).

[12] J. P. Bouchaud, J. Phys. I 2, 1705 (1992).

[13] R. Metzler, J. Jae-Hyung, A. Cherstvy, and E. Barkai, Phys. Chem. Chem. Phys. 16, 24128 (2014).

[14] J. Klafter and R. Silbey, Phys. Rev. Lett. 44, 55 (1980).

[15] R. Metzler and J. Klafter, Phys. Rep. 339, 1 (2000).

[16] G. Bel and E. Barkai, Phys. Rev. Lett. 94, 240602 (2005).

[17] Y. He, S. Burov, R. Metzler, and E. Barkai, Phys. Rev. Lett. 101, 058101 (2008).

[18] T. Miyaguchi and T. Akimoto, Phys. Rev. E 91, 010102(R) (2015).

[19] T. Miyaguchi and T. Akimoto, Phys. Rev. E 83, 031926 (2011).

[20] C. Manzo, J. A. Torreno-Pina, P. Massignan, G. J. Lapeyre, M. Lewenstein, and M. F. García-Parajo, Phys. Rev. X 5, 011021 (2015).

[21] Supplementary material.

[22] H. Scher and M. Lax, Phys. Rev. B 7, 4502 (1973).

[23] M. Dentz, P. Gouze, A. Russian, J. Dweik, and F. Delay, Adv. Water Resour. 49, 13 (2012).

[24] M. Abramowitz and I. A. Stegun, Handbook of Mathematical Functions (Dover Publications, New York, 1972). 


\title{
Supplementary Material: Self-Averaging and Ergodicity of Anomalous Diffusion in Quenched Random Media
}

\author{
Marco Dentz \\ Spanish National Research Council (IDAEA-CSIC), 08034 Barcelona, Spain* \\ Anna Russian and Philippe Gouze \\ Géosciences, Université de Montpellier 2, CNRS, Montpellier, France
}

(Dated: February 23, 2016)

\begin{abstract}
This supplementary material provides details on the derivation of the expressions for the mean square displacement $m(t)$, its average $\bar{m}(t)$ and variance $\sigma_{m}^{2}(t)$.
\end{abstract}




\section{OBSERVABLES}

\section{Mean Square Displacement}

The mean square displacement in a single medium realization it given by

$$
m(t)=2 \int_{0}^{t} \mathrm{~d} t^{\prime} \int_{0}^{t} d t^{\prime \prime}\left\langle\sqrt{D\left[\mathbf{x}\left(t^{\prime}\right)\right] D\left[\mathbf{x}\left(t^{\prime \prime}\right)\right]} \boldsymbol{\xi}\left(t^{\prime}\right) \boldsymbol{\xi}\left(t^{\prime \prime}\right)\right\rangle=2 d \int_{0}^{t} d t^{\prime}\left\langle D\left[\mathbf{x}\left(t^{\prime}\right)\right]\right\rangle .
$$

Note that we use the Ito interpretation such that $\langle D[\mathbf{x}(t)] \boldsymbol{\xi}(t)\rangle=0$. Thus, the second equal sign follows because the only non-vanishing correlation is the one between $\boldsymbol{\xi}\left(t^{\prime}\right)$ and $\boldsymbol{\xi}\left(t^{\prime \prime}\right)$. This can be seen as follows. Suppose that there is a correlation between $\sqrt{D\left[\mathbf{x}\left(t^{\prime}\right)\right]}$ and $\boldsymbol{\xi}\left(t^{\prime \prime}\right)$. This means that $t^{\prime}>t^{\prime \prime}$. Then however, the correlation between $\sqrt{D\left[\mathbf{x}\left(t^{\prime \prime}\right)\right]}$ and $\boldsymbol{\xi}\left(t^{\prime}\right)$ vanishes. And the same holds vice versa.

We set now $D(\mathbf{x})=\kappa / \theta(\mathbf{x})$ and define $\mathrm{d} s=\mathrm{d} t / \theta[\mathbf{x}(t)]$, which gives for $m(t)$

$$
m(t)=2 \kappa d\langle s(t)\rangle=d \ell^{2}\left\langle n_{t}\right\rangle
$$

For the last equation, we used the coarse grained particle trajectory

$$
\mathbf{x}_{n+1}=\mathbf{x}_{n}+\ell \boldsymbol{\eta}_{n+1}, \quad t_{n+1}=t_{n}+\theta\left(\mathbf{x}_{n}\right) \hat{\tau},
$$

where $\hat{\tau}$ is an exponential random variable with mean $\tau_{\kappa}=\ell^{2} /(2 \kappa)$. Expression (2) can be further expanded as

$$
m(t)=d \ell^{2}\left\langle n_{t}\right\rangle=d \ell^{2} \sum_{n=0}^{\infty} n\left\langle\delta_{n, n_{t}}\right\rangle=d \ell^{2} \sum_{n=0}^{\infty} n\left\langle\mathbb{I}\left(t_{n} \leq t<t_{n+1}\right)\right\rangle .
$$

The noise average may be written as

$$
m(t)=d \ell^{2} \sum_{n=0}^{\infty} \frac{n}{B_{n}} \sum_{i=1}^{B_{n}} \mathbb{I}\left[t_{n}^{(i)} \leq t<t_{n+1}^{(i)}\right], \quad B_{n}=\left(\begin{array}{c}
V_{n} \\
S_{n}
\end{array}\right) .
$$

where $V_{n}=(2 n)^{d / 2}$ is the average number of different sites explored by the random walkers after $n$ steps. The number of independent sites that constitute $t_{n}$ is equal to $S_{n}$. Thus the number of copies of $t_{n}$ at $n$ steps is equal to $B_{n}$, the binomial coefficient of $V_{n}$ over $S_{n}$. Within these $B_{n}$ replica of $t_{n}$ we distinguish $B_{n} / R_{n}$ families of independent members, where $R_{n}=V_{n} / S_{n}$ is the number of members. Again, the total number of explored sites is equal 
to $V_{n}$, therefore the number of independent replica of $t_{n}$ that can be formed is equal to $R_{n}$. We can write (5) now as

$$
m(t)=d \ell^{2} \sum_{n=0}^{\infty} \frac{n R_{n}}{B_{n}} \sum_{i=1}^{B_{n} / R_{n}}\left\{\frac{1}{R_{n}} \sum_{k=1}^{R_{n}} \mathbb{I}\left[t_{n}^{(i, k)} \leq t<t_{n+1}^{(i, k)}\right]\right\} .
$$

Note that the expression in curly brackets denotes the distribution of the number of steps needed to reach time $t$ in the section of the medium sampled by the random walker. As each of the $B_{n} / R_{n}$ families explores in average the same disorder, they are in average the same. Thus, we approximate

$$
m(t) \approx d \ell^{2} \sum_{n=0}^{\infty} \frac{n}{R_{n}} \sum_{k=1}^{R_{n}} \mathbb{I}\left[t_{n}^{(k)} \leq t<t_{n+1}^{(k)}\right] .
$$

We argue that this expression captures the impact of the noise on the random walkers' sampling of the medium heterogeneity in a single realization adequately.

\section{Time Averaged Mean Square Displacement}

The time averaged mean square displacement is defined as

$$
m_{\Delta}(t)=\frac{1}{t-\Delta} \int_{0}^{t-\Delta} \mathrm{d} t^{\prime}\left[\mathbf{x}\left(t^{\prime}+\Delta\right)-\mathbf{x}\left(t^{\prime}\right)\right]^{2}
$$

The increment $\mathbf{x}_{\Delta}(t)=\mathbf{x}(t+\Delta)-\mathbf{x}(t)$ can be written as

$$
\mathbf{x}_{\Delta}(t)=\int_{t}^{t+\Delta} \mathrm{d} t^{\prime} \sqrt{2 D\left[\mathbf{x}\left(t^{\prime}\right)\right]} \boldsymbol{\xi}\left(t^{\prime}\right)
$$

We consider the limit of $\Delta \rightarrow 0$, for which we obtain

$$
\mathbf{x}_{\Delta}(t)=\sqrt{2 D[\mathbf{x}(t)]} \boldsymbol{w}_{\Delta}(t), \quad \boldsymbol{w}_{\Delta}(t)=\int_{t}^{t+\Delta} \mathrm{d} t^{\prime} \boldsymbol{\xi}\left(t^{\prime}\right),
$$

with $\left\langle\boldsymbol{w}_{\Delta}(t)\right\rangle=\mathbf{0}$ and $\left\langle\boldsymbol{w}_{\Delta}(t) \cdot \boldsymbol{w}_{\Delta}\left(t^{\prime}\right)\right\rangle$ equal to $d \Delta$ if $\left|t-t^{\prime}\right|<\Delta$ and 0 else. This gives for $m_{\Delta}(t)$

$$
m_{\Delta}(t)=\frac{2}{t} \int_{0}^{t} \mathrm{~d} t^{\prime} D\left[\mathbf{x}\left(t^{\prime}\right)\right] \boldsymbol{w}_{\Delta}\left(t^{\prime}\right)^{2}
$$


The ergodicity of the diffusion process in a single disorder realization is probed by the variance of $m_{\Delta}(t)$ with respect to the noise ensemble. The noise mean of $m_{\Delta}(t)$ is given by

$$
\left\langle m_{\Delta}(t)\right\rangle=\frac{2 d \Delta}{t}\left\langle\int_{0}^{t} \mathrm{~d} t^{\prime} D\left[\mathbf{x}\left(t^{\prime}\right)\right]\right\rangle=\frac{m(t) \Delta}{t} .
$$

For the noise averaged mean squared $m_{\Delta}(t)$, we obtain

$$
\left\langle m_{\Delta}(t)^{2}\right\rangle=\frac{4}{t^{2}} \int_{0}^{t} \mathrm{~d} t^{\prime} \int_{0}^{t} \mathrm{~d} t^{\prime \prime} D\left[\mathbf{x}\left(t^{\prime}\right)\right] D\left[\mathbf{x}\left(t^{\prime \prime}\right)\right]\left\langle w_{\Delta}\left(t^{\prime}\right)^{2} w_{\Delta}\left(t^{\prime \prime}\right)^{2}\right\rangle=\frac{m(t)^{2} \Delta^{2}}{t^{2}}+\ldots,
$$

where the dots denote subleading contributions of order $\Delta^{3} / t^{3}$. Thus, diffusion is of course ergodic in a single medium realization.

\section{Average Mean Square Displacement}

The average mean square displacement is obtained from (2) and (4) as

$$
\bar{m}(t)=d \ell^{2} \overline{\left\langle n_{t}\right\rangle}=d \ell^{2} \sum_{n=0}^{\infty} n \overline{\mathbb{I}\left(t_{n} \leq t<t_{n+1}\right)} .
$$

\section{Disorder Variance of Mean Square Displacement}

The second disorder moment of $m(t)$ reads as

$$
\overline{m(t)^{2}}=\sum_{n, k=0}^{\infty} n k d^{2} \ell^{4} \frac{1}{R_{n}} \frac{1}{R_{k}} \sum_{i=1}^{R_{n}} \sum_{j=1}^{R_{k}} \overline{\mathbb{I}\left[t_{n}^{(i)} \leq t<t_{n+1}^{(i)}\right] \mathbb{I}\left[t_{k}^{(j)} \leq t<t_{k+1}^{(j)}\right]} .
$$

By definition, the $t_{n}^{(i)}$ and $t_{k}^{(j)}$ are independent for $i \neq j$. For $i=j$ we have by virtue of the impulse functions that $n=k$. Thus, we can write $\overline{m(t)^{2}}$ as

$$
\begin{aligned}
& \left.\overline{m(t)^{2}}=d^{2} \ell^{4} \sum_{n=0}^{\infty} \frac{n^{2}}{R_{n}} \overline{\mathbb{I}\left(t_{n} \leq t<t_{n+1}\right.}\right)-2 d^{2} \ell^{4} \sum_{n>k=0}^{\infty} \frac{n k}{R_{n}} \overline{\mathbb{I}\left(t_{n} \leq t<t_{n+1}\right)} \overline{\mathbb{I}\left(t_{k} \leq t<t_{k+1}\right)} \\
& -d^{2} \ell^{4} \sum_{n=0}^{\infty} \frac{n^{2}}{R_{n}} \overline{\mathbb{I}\left(t_{n} \leq t<t_{n+1}\right)^{2}}+\sum_{n=0}^{\infty} n d \ell^{2} \overline{\mathbb{I}\left(t_{n} \leq t<t_{n+1}\right)} \sum_{k=0}^{\infty} k d \ell^{2} \overline{\mathbb{I}\left(t_{k} \leq t<t_{k+1}\right)}
\end{aligned}
$$

Note that the last expression on the right side is equal to the square of the average second moment. Thus, we obtain for the variance of the mean square displacement the expression

$$
\begin{aligned}
\sigma_{m}^{2}(t) & =d^{2} \ell^{4} \sum_{n=0}^{\infty} \frac{n^{2}}{R_{n}} \overline{\mathbb{I}\left(t_{n} \leq t<t_{n+1}\right)}-2 d^{2} \ell^{4} \sum_{n>k=0}^{\infty} \frac{n k}{R_{n}} \overline{\mathbb{I}\left(t_{n} \leq t<t_{n+1}\right)} \overline{\mathbb{I}\left(t_{k} \leq t<t_{k+1}\right)} \\
& -d^{2} \ell^{4} \sum_{n=0}^{\infty} \frac{n^{2}}{R_{n}} \overline{\mathbb{I}\left(t_{n} \leq t<t_{n+1}\right)}
\end{aligned}
$$




\section{EXPLICIT EXPRESSIONS AND SCALING BEHAVIORS}

First, we consider the Laplace transform of the transition time distribution for times $t \gg \tau_{\kappa}$. As indicated in the main text, the transition time distribution is heavy tailed such. It behaves as

$$
\psi(\tau) \sim\left(\frac{\tau}{\tau_{\kappa}}\right)^{-1-\beta},
$$

where we focus on the range $0<\beta<1$. It is well known that its Laplace transform can be expanded as

$$
\psi(\lambda)=1-a_{\beta} \lambda^{\beta}+\ldots
$$

for $\lambda \tau_{\kappa} \ll 1$ and $a_{\beta}$ a constant. Note that the numerical simulations consider a Pareto distribution of $\theta, p_{\theta}(\theta)=\beta \theta^{-1-\beta}$ for $\theta>1$ which gives for the distribution of transition times $\psi(\tau)$ the explicit expression

$$
\psi(\tau)=\frac{\beta}{\tau_{\kappa}}\left(\frac{\tau}{\tau_{\kappa}}\right)^{-1-\beta} \gamma\left(1+\beta, \tau / \tau_{\kappa}\right),
$$

with $\gamma(a, z)$ the lower incomplete Gamma function [1].

In order to determine now the scaling behaviors of the mean and variance of the mean square displacement, we consider the average over the impulse function and its scaling form,

$$
I_{n}(t)=\overline{\mathbb{I}\left(t_{n} \leq t<t_{n+1}\right)}
$$

Note that $I_{n}(t)$ is equal to the probability distribution of the $n_{t}$. Its Laplace transform is given by

$$
I_{n}(\lambda)=\lambda^{-1} \overline{\left[\exp \left(-\lambda t_{n}\right)-\exp \left(-\lambda t_{n+1}\right)\right]}
$$

The times $t_{n}$ and $t_{n+1}$ are given by

$$
t_{n}=\sum_{i=1}^{S_{n}} \gamma_{n} \tau_{i}, \quad t_{n+1}=\sum_{i=1}^{S_{n+1}} \gamma_{n+1} \tau_{i}
$$

where we define $\gamma_{n} \equiv n / S_{n}$. Using these expressions in (22) and performing the ensemble average we obtain

$$
I_{n}(\lambda)=\lambda^{-1}\left[\psi\left(\lambda \gamma_{n}\right)^{S_{n}}-\psi\left(\lambda \gamma_{n+1}\right)^{S_{n+1}}\right]
$$


Using (19) and by virtue of the generalized central limit theorem, we approximate now

$$
f_{n}(\lambda) \equiv \psi\left(\lambda \gamma_{n}\right)^{S_{n}} \approx \exp \left[-S_{n} a_{\beta}\left(\lambda \gamma_{n}\right)^{\beta}\right]
$$

which in time domain has the scaling form

$$
f_{n}(t)=\frac{1}{\alpha_{n}} f_{\beta}\left(t / \alpha_{n}\right)
$$

where we define for convenience $\alpha_{n}=\gamma_{n} S_{n}^{1 / \beta}$.

We define now $F_{n}(\lambda)=\lambda^{-1} f_{n}(\lambda)$ such that $I_{n}(\lambda)$ can be written as

$$
I_{n}(\lambda)=F_{n}(\lambda)-F_{n+1}(\lambda)
$$

The $F_{n}(t)$ has the scaling form

$$
F_{n}(t)=F_{\beta}\left(t / \alpha_{n}\right)=\int_{0}^{t / \alpha_{n}} d t^{\prime} f_{\beta}\left(t^{\prime}\right)
$$

We approximate now $I_{n}(t)$ as

$$
I_{n}(t) \approx-\frac{\mathrm{d} F_{n}(t)}{\mathrm{d} n}=\frac{t}{\alpha_{n}} \frac{\mathrm{d} \ln \left(\alpha_{n}\right)}{\mathrm{d} n} f_{\beta}\left(t / \alpha_{n}\right) .
$$

Note that the Laplace transform of $f_{\beta}(\lambda)$ is according to (25) and (26) given by

$$
f_{\beta}(\lambda)=\exp \left(-a_{\beta} \lambda^{\beta}\right)
$$

\section{Average Mean Square Displacement}

We can write (14) using (29) as

$$
\bar{m}(t) \approx d \ell^{2} \int_{0}^{\infty} \mathrm{d} n \frac{n t}{\alpha_{n}} \frac{\mathrm{d} \ln \left(\alpha_{n}\right)}{\mathrm{d} n} f_{\beta}\left(t / \alpha_{n}\right)=d \ell^{2} \bar{n}_{t}
$$

In $d<2, S_{n} \sim n^{d / 2}$ and $\gamma_{n}=n^{1-d / 2}$, therefore $\alpha_{n}=n^{\frac{2 \beta-d \beta+d}{2 \beta}}$ such that

$$
\bar{m}(t) \approx d \ell^{2} \frac{2 \beta-d \beta+d}{2 \beta} \int_{0}^{\infty} \mathrm{d} n \frac{t}{n^{\frac{2 \beta-d \beta+d}{2 \beta}}} f_{\beta}\left(\frac{t}{n^{\frac{2 \beta-d \beta+d}{2 \beta}}}\right) \propto t^{\frac{2 \beta}{2 \beta-d \beta+d}} .
$$

Thus, we have at the same time that $\bar{n}_{t} \propto t^{\frac{2 \beta}{2 \beta-d \beta+d}}$. 
In $d=2, S_{n} \sim n / \ln (n)$ and $\gamma_{n}=\ln (n)$, therefore $\alpha_{n}=n^{1 / \beta} \ln (n)^{1-1 / \beta}$ such that

$$
\bar{m}(t) \approx \frac{d \ell^{2}}{\beta} \int_{0}^{\infty} \mathrm{d} n \frac{t}{n^{1 / \beta} \ln (n)^{1-1 / \beta}} f_{\beta}^{\prime}\left(\frac{t}{n^{1 / \beta} \ln (n)^{1-1 / \beta}}\right) \propto t^{\beta} \ln (t)^{1-\beta} .
$$

Thus, we have at the same time that $\bar{n}_{t} \propto t^{\beta} \ln (t)^{1-\beta}$.

In $d=3, S_{n} \sim n$ and $\gamma_{n}=1$, therefore $\alpha_{n}=n^{1 / \beta}$ such that

$$
\bar{m}(t) \approx \frac{d \ell^{2}}{\beta} \int_{0}^{\infty} \mathrm{d} n \frac{t}{n^{1 / \beta}} f_{\beta}\left(\frac{t}{n^{1 / \beta}}\right) \propto t^{\beta} .
$$

\section{Variance of the Mean Square Displacement}

We split expression (17) into three parts,

$$
\sigma_{m}^{2}(t)=d^{2} \ell^{4} J_{1}(t)-d^{2} \ell^{4} J_{2}(t)-d^{2} \ell^{4} J_{3}(t)
$$

where we defined

$$
\begin{aligned}
& \left.J_{1}(t)=\sum_{n=0}^{\infty} \frac{n^{2}}{R_{n}} \overline{\mathbb{I}\left(t_{n} \leq t<t_{n+1}\right.}\right) \\
& J_{2}(t)=2 \sum_{n>k=0}^{\infty} \frac{n k}{R_{n}} \overline{\mathbb{I}\left(t_{n} \leq t<t_{n+1}\right)} \overline{\mathbb{I}\left(t_{k} \leq t<t_{k+1}\right)} \\
& J_{3}(t)=\sum_{n=0}^{\infty} \frac{n^{2}}{R_{n}} \overline{\mathbb{I}\left(t_{n} \leq t<t_{n+1}\right)} .
\end{aligned}
$$

For $J_{1}(t)$, we obtain immediately by using $(29)$

$$
J_{1}(t) \approx \int_{0}^{\infty} \mathrm{d} n \frac{n^{2}}{R_{n}} \frac{t}{\alpha_{n}} \frac{\mathrm{d} \ln \left(\alpha_{n}\right)}{\mathrm{d} n} f_{\beta}\left(t / \alpha_{n}\right) .
$$

In $d<2, S_{n} \sim n^{d / 2}$ and $\gamma_{n}=n^{1-d / 2}$, therefore $\alpha_{n}=n^{\frac{2 \beta-d \beta+d}{2 \beta}}$ and $R_{n}=2^{d / 2}$ such that

$$
J_{1}(t) \approx \frac{2 \beta-d \beta+d}{2^{1+d / 2} \beta} \int_{0}^{\infty} \mathrm{d} n n \frac{t}{n^{\frac{2 \beta-d \beta+d}{2 \beta}}} f_{\beta}\left(\frac{t}{n^{\frac{2 \beta-d \beta+d}{2 \beta}}}\right) \propto t^{\frac{4 \beta}{2 \beta-d \beta+d}} .
$$

In $d=2, S_{n} \sim n / \ln (n)$ and $\gamma_{n}=\ln (n)$, therefore $\alpha_{n}=n^{1 / \beta} \ln (n)^{1-1 / \beta}$, and $R_{n}=2 \ln (n)$ such that

$$
J_{1}(t) \approx \frac{1}{2 \beta} \int_{0}^{\infty} \mathrm{d} n \frac{n}{\ln (n)} \frac{t}{n^{1 / \beta} \ln (n)^{1-1 / \beta}} f_{\beta}^{\prime}\left(\frac{t}{n^{1 / \beta} \ln (n)^{1-1 / \beta}}\right) \propto t^{2 \beta} \ln (t)^{1-2 \beta} .
$$


In $d=3, S_{n} \sim n$ and $\gamma_{n}=1$, therefore $\alpha_{n}=n^{1 / \beta}$, and $R_{n}=2^{d / 2} n^{d / 2-1}$ such that

$$
J_{1}(t) \approx \frac{1}{2^{d / 2} \beta} \int_{0}^{\infty} \mathrm{d} n n^{2-d / 2} \frac{t}{n^{1 / \beta}} f_{\beta}\left(\frac{t}{n^{1 / \beta}}\right) \propto t^{\beta(3-d / 2)} .
$$

For $J_{2}(t)$, we obtain by using $(29)$

$$
J_{2}(t) \approx 2 \int_{0}^{\infty} \mathrm{d} n \frac{n}{R_{n}} \frac{t}{\alpha_{n}} \frac{\mathrm{d} \ln \left(\alpha_{n}\right)}{\mathrm{d} n} f_{\beta}\left(t / \alpha_{n}\right) \int_{0}^{n} \mathrm{~d} k k \frac{t}{\alpha_{k}} \frac{\mathrm{d} \ln \left(\alpha_{k}\right)}{\mathrm{d} k} f_{\beta}\left(t / \alpha_{k}\right) .
$$

In $d<2, S_{n} \sim n^{d / 2}$ and $\gamma_{n}=n^{1-d / 2}$, therefore $\alpha_{n}=n^{\frac{2 \beta-d \beta+d}{2 \beta}}$ and $R_{n}=2^{d / 2}$ such that

$$
\begin{aligned}
J_{2}(t) & \approx \frac{(2 \beta-d \beta+d)^{2}}{2^{1+d / 2} \beta^{2}} \int_{0}^{\infty} \mathrm{d} n \frac{t}{n^{\frac{2 \beta-d \beta+d}{2 \beta}}} f_{\beta}\left(\frac{t}{n^{\frac{2 \beta-d \beta+d}{2 \beta}}}\right) \int_{0}^{n} \mathrm{~d} k \frac{t}{k^{\frac{2 \beta-d \beta+d}{2 \beta}}} f_{\beta}\left(\frac{t}{k^{\frac{2 \beta-d \beta+d}{2 \beta}}}\right) \\
& \propto t^{\frac{4 \beta}{2 \beta-d \beta+d}} .
\end{aligned}
$$

In $d=2$, we obtain along the same lines that

$$
J_{2}(t) \propto t^{2 \beta} \ln (t)^{1-2 \beta}
$$

and for $d>2$, we find that

$$
J_{2}(t) \propto t^{\beta(3-d / 2)}
$$

For $J_{3}(t)$, we obtain by using (29),

$$
J_{3}(t) \approx \int_{0}^{\infty} \mathrm{d} n \frac{n^{2}}{R_{n}}\left[\frac{t}{\alpha_{n}} \frac{\mathrm{d} \ln \left(\alpha_{n}\right)}{\mathrm{d} n} f_{\beta}\left(t / \alpha_{n}\right)\right]^{2}
$$

In $d<2, S_{n} \sim n^{d / 2}$ and $\gamma_{n}=n^{1-d / 2}$, therefore $\alpha_{n}=n^{\frac{2 \beta-d \beta+d}{2 \beta}}$ and $R_{n}=2^{d / 2}$ such that

$$
J_{3}(t) \approx \frac{(2 \beta-d \beta+d)^{2}}{2^{2+d / 2} \beta^{2}} \int_{0}^{\infty} \mathrm{d} n\left[\frac{t}{n^{\frac{2 \beta-d \beta+d}{2 \beta}}} f_{\beta}\left(\frac{t}{n^{\frac{2 \beta-d \beta+d}{2 \beta}}}\right)\right]^{2} \propto t^{\frac{2 \beta}{2 \beta-d \beta+d}} .
$$

In $d=2$, we obtain along the same lines that

$$
J_{3}(t) \propto\left(\frac{t}{\ln (t)}\right)^{\beta}
$$

and for $d=3$, we find that

$$
J_{3}(t) \propto t^{\beta(1-d / 2)}
$$


Thus the leading contributions to $\sigma_{m}^{2}(t)$ at long times come from $J_{1}(t)$ and $J_{2}(t)$.

* Electronic address: marco.dentz@csic.es

[1] M. Abramowitz and I. A. Stegun, Handbook of Mathematical Functions (Dover Publications, New York, 1972). 